\title{
Association between Total Cholesterol and the Direct to Total Bilirubin Ratio in Serum
}

\author{
Masataka Mine, $\mathrm{MD}^{1)}$, Nobuyuki Masaki, MD²), Tetsuya Hisada, $\mathrm{MD}^{3)}$, \\ Bonpei Takase, $\mathrm{MD}^{2)}$ and Takeshi Adachi, $\mathrm{MD}^{3)}$
}

\begin{abstract}
:
Background: Bilirubin and cholesterol are associated with cardiovascular disease, though the relationship between these metabolites has not been fully investigated. Methods: Healthy subjects were enrolled. Annual cross-sectional assessment (year; 2012, 2016) and retrospective longitudinal assessment (year; 2012 to 2016) were performed. The subjects with receiving statin treatment were analyzed separately. Bilirubin ratio was defined as direct (or conjugated) bilirubin divided by the total bilirubin level in serum. Then, differences among the measurement principles were examined (year; 2017). Results: Bilirubin ratio measured by the vanadate oxidation method (VA) was strongly correlated with the total cholesterol level in both the non-statin group (2012: $\mathrm{r}=-0.753, \mathrm{n}=740 ; 2016: \mathrm{r}=-0.763, \mathrm{n}=792$; all $\mathrm{p}<0.001)$ and the statin group (2012: $\mathrm{r}=-0.825, \mathrm{n}=39 ; 2016: \mathrm{r}=-0.855, \mathrm{n}=43$; all $\mathrm{p}<0.001$ ). The percent change of the bilirubin ratio from 2012 to 2016 was negatively proportional to that of total cholesterol $(\mathrm{r}=-0.634, \mathrm{n}=538 ; \mathrm{p}<0.001)$. A comparison of the two different methods in 305 participants in 2017 showed that the correlation was stronger with the VA than with the bilirubin oxidase method (BOD), (VA: $r=-0.759, p<0.001$; BOD: $r=-0.160, p=0.005$ ). In a laboratory experiment, the addition of cholesterol to human serum reduced the bilirubin ratio in both methods. Conclusion: A strong association between cholesterol and the ratio of direct to total bilirubin was revealed.
\end{abstract}

\section{Key words:}

Metabolism, Bilirubin, Cholesterol, Clinical studies

\section{Introduction}

Bilirubin is the end product of heme catabolism in the turnover of erythrocyte hemoglobin and a potent endogenous antioxidant ${ }^{1,2}$. The clinical importance of bilirubin in metabolic and cardiovascular disease has recently been acknowledged ${ }^{3,4}$. Mild bilirubinemia in Gilbert's syndrome caused by a polymorphism (TA repeat) in the hepatic uridine diphosphate glucuronosyl transferase 1A1 (UGT1A 1) gene is thought to be protective against cardiovascular disease $^{5,6)}$. Furthermore, in studies among the general population, low bilirubin concentrations are associated with smoking and drinking ${ }^{7}$, high oxidative stress $^{8}$, dyslipidemia ${ }^{9-11}$, metabolic syndrome ${ }^{12-17}$, cardiovascular disease ${ }^{18-21)}$, and mor- tality ${ }^{22)}$. However, the relationship becomes more obscure in aging populations because the prevalence of bilirubinemia increases due to hepatic dysfunction. The curve for overall risk ratio for both cardiovascular disease ${ }^{19)}$ and mortality ${ }^{22}$ versus bilirubin is U-shaped. Nevertheless, apart from genetic disorders, the exact reason behind the higher bilirubin levels in people with low cardiovascular risk is unknown.

Thus, numerous studies in the cardiovascular field have reported the significance of bilirubin. However, there are several limitations to the evaluation of bilirubin. First, total bilirubin level can be changed in conditions such as caloric restriction and exercise ${ }^{23}$. Second, there are two definitions used to evaluate hepatobiliary functions including bilirubin glucuronidation and biliary efflux: "direct" and "conjugated"

1) Japan Air Self-Defense Force Aeromedical Laboratory, Sayama, Japan

2) Department of Intensive Care Medicine, National Defense Medical College, Tokorozawa, Japan

3) Department of Cardiology, National Defense Medical College, Tokorozawa, Japan

Corresponding author: Nobuyuki Masaki, MD, masakinobuyuki@ hotmail.com

Received: December 16, 2019, Accepted: February 20, 2020

Copyright (C) 2019 Japan Society for Vascular Failure 
bilirubin. The delta fraction in chromatography (delta bilirubin) is a result of the substitution of albumin for the glucuronide moiety of conjugated bilirubin. The delta bilirubin is included in the "direct" bilirubin measured by the diazo method or vanadate oxidation method (VA) but excluded in the "conjugated" bilirubin measured by the bilirubin oxidase method (BOD).

Hence, the evaluation of bilirubin levels is difficult. The bilirubin ratio (direct to total bilirubin) adjusts for day-today variance in the total bilirubin level and may be more sensitive to interaction with metabolic and cardiovascular risks. This ratio is traditionally used to classify hyperbilirubinemia $^{24)}$ and to evaluate the severity of fulminant hepati$\mathrm{tis}^{25}$. Thus, this study investigated the relationship between bilirubin ratio and serum cholesterol levels using longitudinal data from healthy middle-aged individuals collected over 5 years. Subsequently, we speculated regarding the reason for the discovered correlations.

\section{Materials and Methods}

\section{Study design and population}

We retrospectively analyzed the data of Japanese subjects who agreed to participate in a survey on metabolic syndrome at Iruma Air Defense Force Base (Saitama, Japan) and to have their bilirubin levels measured between 2012 and 2017. This clinical study conformed to the ethical guidelines of the Declaration of Helsinki and was approved by the ethics committee at our institute. Written informed consent was obtained from all participants. The exclusion criteria were suspected inherited hyperbilirubinemia, and a history of cholecystectomy. Cross-sectional analyses were performed on annual datasets. A longitudinal analysis of the subjects followed-up throughout the 5 years was performed.

\section{Anthropometric and biochemical data}

Data were collected at a single facility. Sociodemographic information, such as age and sex, were collected from the database before the examinations. Weight and height were measured using standard methods and digitally recorded. Chest circumferences were measured just below the nipples. Waist circumferences were measured at the navel as participants exhaled lightly while standing. Blood pressure was measured around the upper arm with participants in a sitting position after a few minutes of rest. Life history, including alcohol intake, smoking, and medications, was investigated by a questionnaire. Fasting blood samples were taken in the morning on the day of examination. All participants were asked to fast from the previous evening. Blood specimens were immediately processed at the same facility.

Total bilirubin and direct bilirubin levels were measured by the VA from 2012 to 2017 (Total Bilirubin E-HA, Direct Bilirubin E-HA, Wako Pure Chemical Industries Ltd., Osaka, Japan) and doubly measured by the BOD in 2017 (Acurus Auto T-BIL, D-BIL, Shino-Test Corp., Tokyo, Ja- pan). We defined the bilirubin ratio as the direct or conjugated bilirubin level divided by the total bilirubin level.

Total cholesterol was measured by the cholesterol oxidase-HMMPS [ $N$-(3-sulfopropyl)-3-methoxy-5-methylaniline] method (L-Type CHO M, Wako Pure Chemical Industries Ltd.) in 2012 and the HDAOS [N-(2-hydroxy-3sulfopropyl)-3, 5-dimethoxyaniline Sodium] method (Quickauto neo T-CHO II, Shino-Test Corp., Tokyo, Japan) in 2016 and 2017. Low-density lipoprotein (LDL) cholesterol was measured by the selective elimination method (L-Type LDLC M, Wako Pure Chemical Industries Ltd. in 2012 and Quick-auto neo LDL-C, Shino-Test Corp. in 2016 and 2017). High-density lipoprotein (HDL) cholesterol was measured by the selective elimination method (L-Type HDL-C M, Wako in 2012 and Quick-auto neo HDL-C, Shino-Test Corp. in 2016 and 2017). Triglycerides were measured by the glycerol blanking method (L-Type Triglyceride M, Wako Pure Chemical Industries Ltd. in 2012 and Quick-auto neo TG II, Shino-Test Corp. in 2016 and 2017).

Non-alcoholic fatty liver disease (NAFLD) was diagnosed by abdominal ultrasound as follows. After skilled laboratory technicians performed a primary assessment, a gastroenterologist conducted a second evaluation and reached a diagnosis. Metabolic syndrome was defined as the presence of three or more of the following five abnormalities: 1) abnormal waist circumference (male $\geq 85 \mathrm{~cm}$, female $\geq 90 \mathrm{~cm}$ ), 2) fasting serum triglycerides $\geq 150 \mathrm{mg} / \mathrm{dL}, 3$ ) $\mathrm{HDL}<40$ $\mathrm{mg} / \mathrm{dL}, 4)$ fasting blood glucose $\geq 110 \mathrm{mg} / \mathrm{dL}$, and 5) blood pressure $\geq 130 / 85 \mathrm{mmHg}^{26}$. The homeostasis model assessment for insulin resistance index and insulin secretion ability were assessed based on fasting insulin levels as previously described $^{27)}$.

\section{Statistics}

Summary data are presented as the mean \pm standard deviation (SD) with a $95 \%$ confidential interval for scale. A percent change $(\%)$ is the difference between the new and original values of a parameter divided by the latter value. Correlation between two variables was assessed using Pearson's method. The paired $t$ test was used to compare lipid data and bilirubin levels before and after the initiation of statin therapy. The overall differences between the three groups in the laboratory examinations were assessed by Friedman's test. The post-hoc analysis between two groups was performed using the Wilcoxon rank sum test. All statistical analyses were performed using SPSS version 22.0 (SPSS Japan, Tokyo). In all analyses, $\mathrm{p}<0.05$ was considered statistically significant.

\section{Results}

\section{Clinical characteristics}

Table 1 shows the background characteristics of the subjects. The number of subjects with/without statin treatment was $39 / 740$ in 2012 and 43/792 in 2016 (Table 1). No sub- 
Table 1. Background characteristics of the subjects

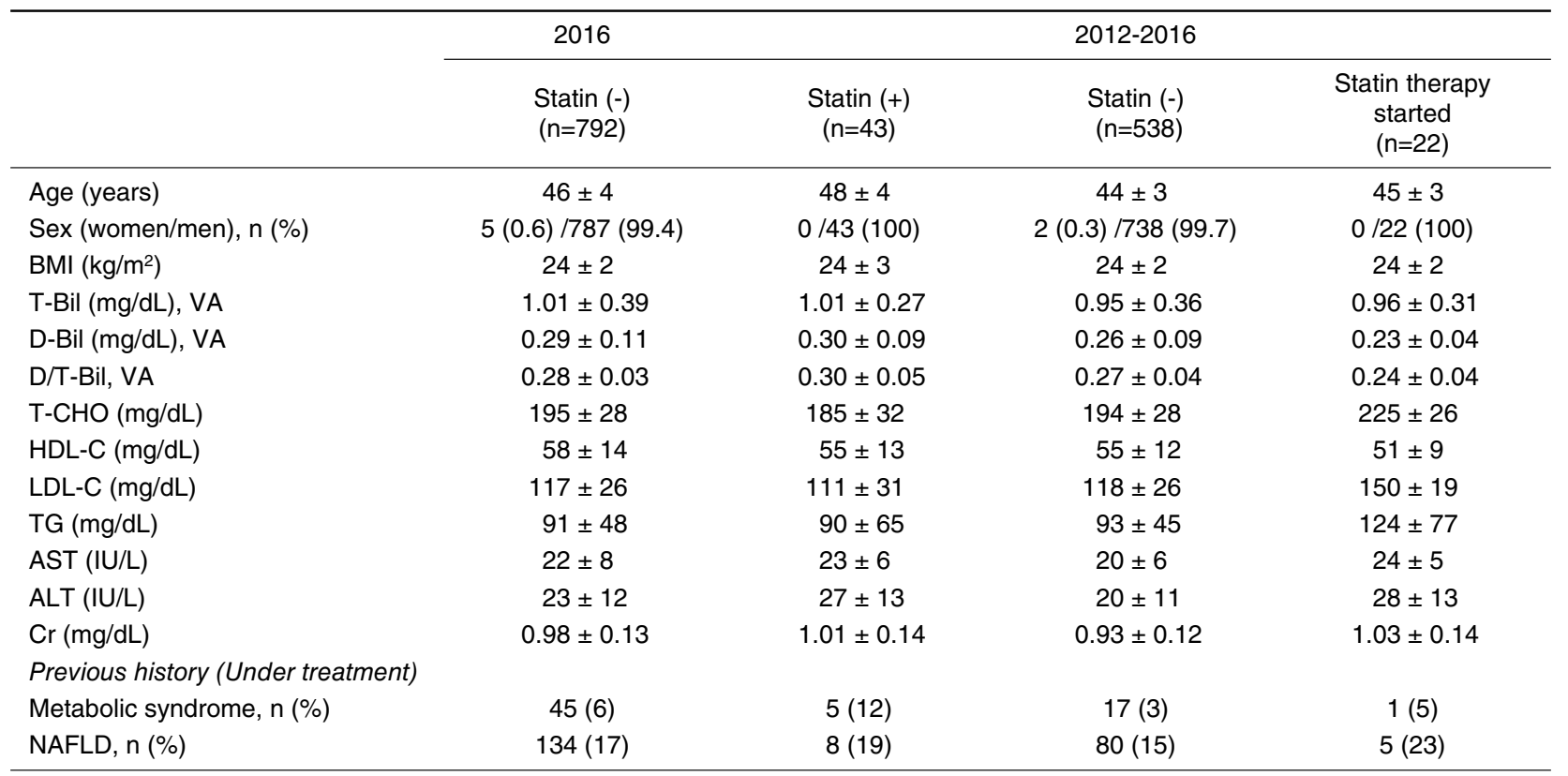

Background characteristics of the participants in 2016 are shown. In a longitudinal observation (2012-2016), 538 subjects did not receive statin treatment, while 22 subjects newly started statin treatment during the study period. The baseline characteristics of these 22 subjects in 2012 are shown in the rightmost column.

VA: vanadate oxidation method, BMI, body mass index; T-Bil, total bilirubin; D-Bil, direct bilirubin; D/T-Bil, bilirubin ratio calculated as direct bilirubin divided by total bilirubin; T-CHO, total cholesterol; HDL-C, high-density lipoprotein cholesterol; LDL-C, low-density lipoprotein cholesterol; TG, triglyceride; AST, aspartate aminotransferase; ALT, alanine aminotransferase; Cr, creatinine kinase; NAFLD, non-alcoholic fatty liver disease.

$n==^{*}: 690, \dagger: 31, \ddagger: 480$.

jects were diagnosed with diabetes mellitus. In the nonstatin group, metabolic syndrome was recognized in $6 \%$ of subjects in 2016. Non-alcoholic fatty liver disease was diagnosed in $17 \%$ of the non-statin group in 2016. In each year, approximately $5 \%$ of subjects received statin therapy. Hence, the study population was characterized as healthy with a very low prevalence of metabolic disorders.

\section{Association between bilirubin ratio and serum total cholesterol level}

There were only weak correlations between the total bilirubin, direct bilirubin, and serum total cholesterol level (Figures 1A and 1B). However, we found a strong inverse relationship between the bilirubin ratio and serum total cholesterol level in each year (2012: $\mathrm{r}=-0.753, \mathrm{n}=740 ; 2016$ : $\mathrm{r}=-0.763, \mathrm{n}=792$; all $\mathrm{p}<0.001$; Figure 1C). The bilirubin ratio correlated negatively with age, body mass index (BMI), systolic blood pressure, LDL cholesterol, and HDL cholesterol in 2016 (Table 2). In subjects receiving statin treatment, the correlation between the bilirubin ratio and serum total cholesterol level was unchanged (2012: $\mathrm{r}=$ $-0.825, \mathrm{n}=39 ; 2016: \mathrm{r}=-0.855, \mathrm{n}=43$; all $\mathrm{p}<0.001$; Figure 1D). Therefore, statins did not affect the relationship between the bilirubin ratios and total cholesterol levels observed in the non-statin group.

\section{Longitudinal assessment of bilirubin ratio}

A longitudinal assessment from 2012 to 2016 was performed for a total of 560 participants $(76 \%)$ from the nonstatin group in 2012 (Table 1). Among them, 538 patients did not receive statin treatment throughout the period, while 22 patients newly started statin treatment. A change in serum total cholesterol level was the strongest contributing factor to a change in the bilirubin ratio $(\mathrm{r}=-0.634, \mathrm{n}=$ 538, $\mathrm{p}<0.001$; Figure 2A).

\section{Effect of statins on the bilirubin ratio}

Twenty-two participants who were not originally receiving statin treatment in 2012 started treatment in 2016 (Table 1). Serum levels of bilirubin, bilirubin ratios, and lipid levels were compared before (2012) and after (2016) statin treatment. Direct bilirubin levels and bilirubin ratios were significantly increased by statin treatment (total bilirubin: 0.96 \pm 0.31 to $1.05 \pm 0.33 \mathrm{mg} / \mathrm{dL}, \mathrm{p}=0.254$; direct bilirubin: $0.23 \pm 0.06$ to $0.31 \pm 0.10 \mathrm{mg} / \mathrm{dL}, \mathrm{p}<0.001$; bilirubin ratio: $0.24 \pm 0.04$ to $0.30 \pm 0.05, \mathrm{p}<0.001)$. Total cholesterol and LDL cholesterol levels decreased, whereas HDL cholesterol level increased (total cholesterol: $224.7 \pm 26.0$ to $185.7 \pm 29.5 \mathrm{mg} / \mathrm{dL}, \mathrm{p}<0.001$; LDL cholesterol: 150.1 \pm 19.4 to $109.2 \pm 31.9 \mathrm{mg} / \mathrm{dL}, \mathrm{p}<0.001$; HDL cholesterol: $50.5 \pm 8.80$ to $55.9 \pm 13.9 \mathrm{mg} / \mathrm{dL}, \mathrm{p}=0.023$; triglycerides: $124.4 \pm 77.0$ to $106.4 \pm 61.7 \mathrm{mg} / \mathrm{dL}, \mathrm{p}<$ 

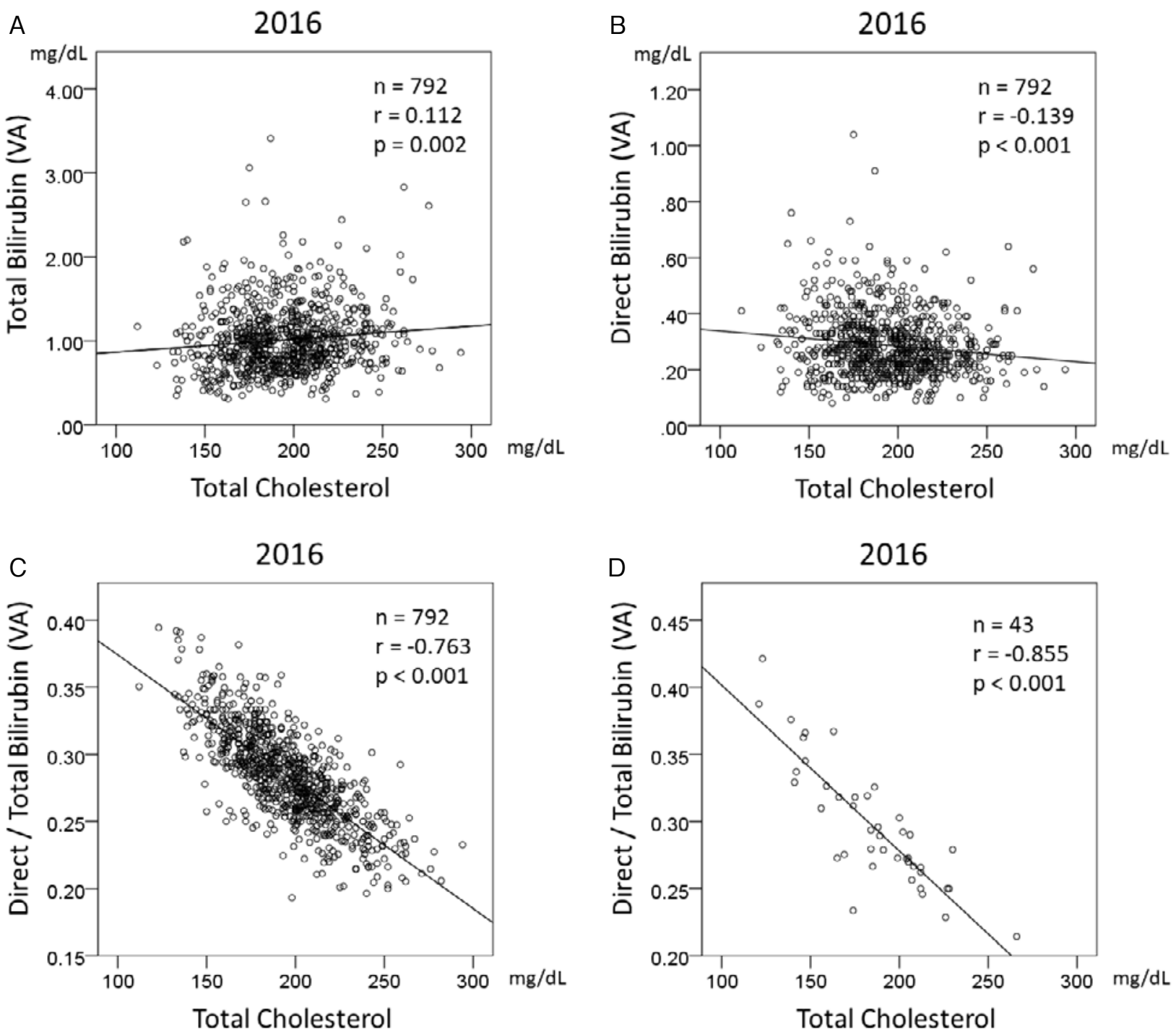

Figure 1. Correlation between the total bilirubin, direct bilirubin, and bilirubin ratios and total cholesterol levels The plots of the two parameters in 2016 are shown [total bilirubin $(A): r=0.112, p=0.002$, direct bilirubin $(B): r=-0.139$, $p<0.001$, bilirubin ratio $(C): r=-0.763, p<0.001 ; n=792$ ]. Correlations between the bilirubin ratios and total cholesterol levels in participants receiving statin treatment in 2016 (D) are also shown $(r=-0.855, n=43 ; p<0.001)$.

VA: vanadate oxidation method.

Table 2. Correlation between bilirubin and clinical parameters

\begin{tabular}{|c|c|c|c|c|c|c|}
\hline \multicolumn{7}{|c|}{$\begin{array}{c}2016 \\
(n=792)\end{array}$} \\
\hline \multirow[t]{2}{*}{ Vanadate Oxidation Method } & \multicolumn{2}{|c|}{ T-Bil } & \multicolumn{2}{|c|}{ D-Bil } & \multicolumn{2}{|c|}{ D/T-Bil } \\
\hline & $r$ & $\mathrm{p}$ & $r$ & $\mathrm{p}$ & $r$ & $p$ \\
\hline Age (years) & 0.077 & 0.030 & 0.031 & 0.378 & -0.129 & $<0.001$ \\
\hline $\mathrm{BMI}\left(\mathrm{kg} / \mathrm{m}^{2}\right)$ & 0.030 & 0.395 & 0.059 & 0.096 & 0.085 & 0.017 \\
\hline T-CHO (mg/dL) & 0.112 & 0.002 & -0.139 & $<0.001$ & -0.763 & $<0.001$ \\
\hline HDL-C (mg/dL) & 0.114 & 0.001 & 0.011 & 0.756 & -0.304 & $<0.001$ \\
\hline LDL-C (mg/dL) & 0.071 & 0.045 & -0.101 & 0.004 & -0.511 & $<0.001$ \\
\hline $\mathrm{TG}(\mathrm{mg} / \mathrm{dL})$ & -0.078 & 0.027 & -0.177 & $<0.001$ & -0.343 & $<0.001$ \\
\hline AST (IU/L) & 0.043 & 0.230 & 0.028 & 0.439 & -0.041 & 0.248 \\
\hline ALT (IU/L) & 0.028 & 0.431 & 0.049 & 0.169 & 0.051 & 0.150 \\
\hline $\mathrm{Cr}(\mathrm{mg} / \mathrm{dL})$ & 0.153 & $<0.001$ & 0.137 & $<0.001$ & -0.026 & 0.470 \\
\hline
\end{tabular}

Abbreviations are the same as in Table 1.

$n=^{*}: 690$. 

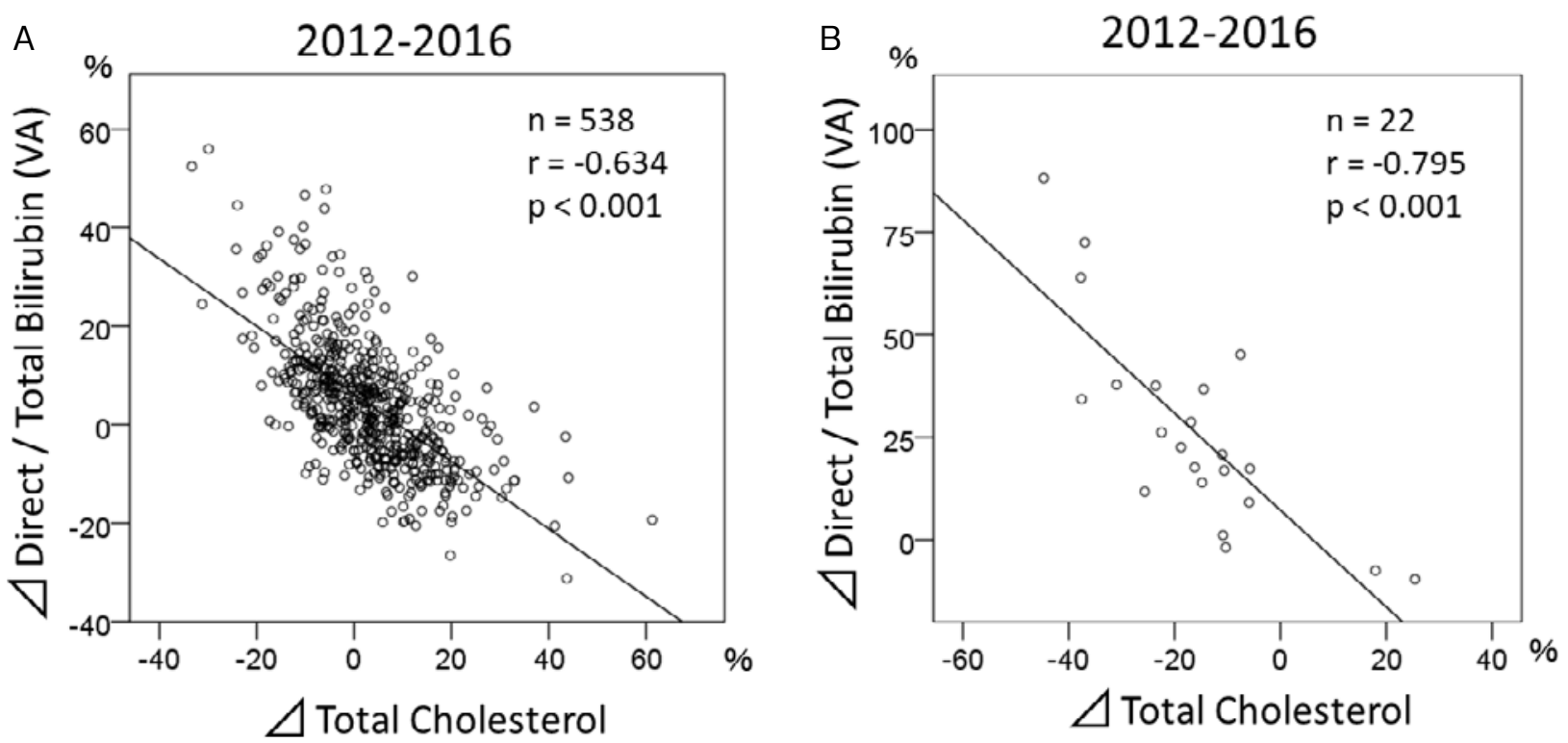

Figure 2. Changes in the bilirubin ratios between 2012 and 2016, before and after statin treatment (A): In non-statin subjects, the percent changes of the bilirubin ratio and serum cholesterol level between 2012 and 2016 correlated negatively with each other. (B): In 22 participants who started statin treatment between 2012 and 2016, the percent change in the bilirubin ratio correlated negatively with that of total cholesterol $(r=-0.795, n=22, p<0.001)$.

0.001). The percent change in the bilirubin ratio correlated negatively with that of total cholesterol $(\mathrm{r}=-0.795, \mathrm{n}=22$, $\mathrm{p}<0.001$; Figure 2B).

\section{The difference in bilirubin ratio between the VA and $B O D$}

We further investigated whether or not the correlation could be observed across different methods of bilirubin measurement. Therefore, 305 participants were additionally enrolled and bilirubin levels were assessed in 2017 using the VA and BOD. The correlation coefficient for total bilirubin level, direct/conjugated bilirubin level, and bilirubin ratio between the methods were $0.995,0.789$, and 0.328 , respectively (all p < 0.001) (Figure 3A-C). However, serum total cholesterol level was more strongly associated with the bilirubin ratio calculated by the VA than with that by the BOD (VA: $\mathrm{r}=-0.759, \mathrm{p}<0.001$; BOD: $\mathrm{r}=-0.160, \mathrm{p}=$ 0.005 ; Figures 3D and 3E).

\section{Discussion}

This study revealed a strong inverse correlation between serum total cholesterol and the ratio of direct to total bilirubin in healthy individuals. In addition, our 5-year longitudinal analysis showed that the percent change of serum total cholesterol correlated inversely with that of the bilirubin ratio. Statin treatment increased the bilirubin ratio but did not change the correlation of the bilirubin ratio with serum total cholesterol. These findings may be attributable to the standardization of direct bilirubin by total bilirubin. Many previous studies reported weak correlations between lipoprotein cholesterol levels and direct bilirubin levels ${ }^{28-30}$. Nevertheless, we suggest that a strong relationship underlies these as-

\section{sociations.}

The exact mechanism underlying the relationship between serum total cholesterol and the bilirubin ratio is unknown. There is a possibility of measurement interference by the following factors. First, the bilirubin ratio was less related to the level of each type of lipoprotein cholesterol than to the total cholesterol level. Second, statin therapy, which inhibits hepatic de-novo cholesterol synthesis, did not change the correlation between the bilirubin ratio and serum total cholesterol level in both the cross-sectional and longitudinal assessments. This result indicates that the bilirubin ratio is not directly associated with de-novo cholesterol synthesis.

We additionally performed the two following examinations. First, we compared two different methods for bilirubin measurement. The correlation between the bilirubin ratio and serum total cholesterol level was significant but less stronger with the BOD than with the VA. The BOD demonstrates only "conjugated" bilirubin ${ }^{31}$. Therefore, the correlation could be attributed to delta bilirubin formed by covalent bonding of conjugated bilirubin to albumin after hepatobiliary secretion ${ }^{32}$. Second, we performed an ex-vivo examination (Supplemental Material). The addition of watersoluble cholesterol decreased direct and conjugated bilirubin when measured with both the VA and BOD. The result was not definitive but suggested a hypothesis that the turbidity of cholesterol decreases the bilirubin ratio.

The VA is less susceptible to hemolysis compared to the classic diazo method ${ }^{33,34)}$. Interference by cholesterol has not been reported so far. Spectrophotometric methods utilize the feature of bilirubin that only direct or conjugated bilirubin can be oxidized to biliverdin in conditions without the additional manipulation of $\mathrm{pH}$ or detergents. The presence of biliverdin decreases the absorbance at $450 \mathrm{nM}$. Therefore, 

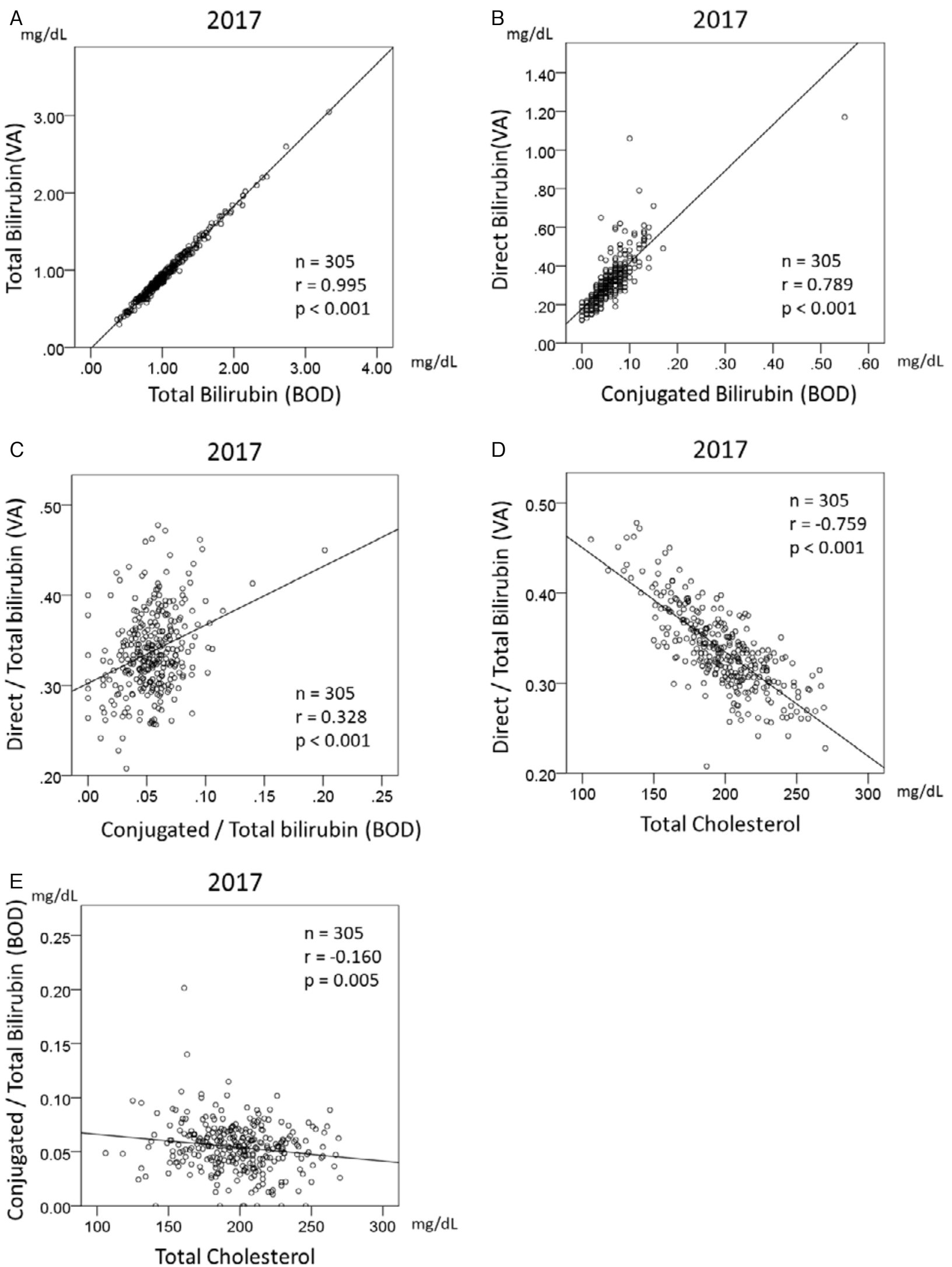

Figure 3. Correlations of the bilirubin measurements with the VA and BOD

$(A)$ : total bilirubin, $(B)$ : direct/conjugated bilirubin, $(C)$ : bilirubin ratio, (D): total cholesterol and direct bilirubin (VA), (E): total cholesterol and conjugated bilirubin (BOD).

VA: vanadate oxidation method, BOD: bilirubin oxidase method. 
high concentrations of cholesterol might affect the process; however, further investigation in this regard is needed. Here, we strongly emphasize that the effect of cholesterol on the absolute value of "direct" or "conjugated" bilirubin is negligible in terms of the diagnosis of cholestasis in clinical practice (Figure 1). Each method has its own merits in the evaluation of hepatobiliary function. Nevertheless, the interpretation of epidemiological studies, especially those assessing direct bilirubin, may be affected due to this.

In addition, our study does not exclude the possibility of direct interaction between bilirubin and cholesterol metabolism within the hepatobiliary system ${ }^{35-37)}$. Bile acids are synthesized by hepatocytes from cholesterol. The molecules have both hydrophilic and highly hydrophobic faces, which facilitate the formation of mixed micelles with bilirubin, phospholipids, and cholesterol ${ }^{38-40)}$. A reduction in hepatic cholesterol may improve the efficiency of bilirubin glucuronidation. The results of one study suggested that the lipid composition in liposomal membranes simulating the endoplasmic reticulum of hepatocytes affects the rate of bilirubin glucuronide formation ${ }^{35}$. Cholesterol-containing membranes decrease the efficiency of glucuronidation. Furthermore, a decrease in cholesterol in hepatocytes could stimulate the biliary efflux of conjugated bilirubin. Statins eliminate bilirubin from the liver by upregulation of multidrug resistance-associated protein (MRP)2 and MRP3, and by suppressing the expression of membrane-associated organic anion transport proteins ${ }^{36}$. However, the bilirubin ratio was not influenced by NAFLD or use of statin in our study. Further investigation is necessary to assess this possibility.

There are several limitations to this study. First, the changes in bilirubin level and bilirubin ratios mediated by statin therapy were assessed via observation of the 22 patients who commenced statin treatment. Thus, further studies are needed to confirm our results. Second, our ex-vivo examination in Supplemental Material was artificial, as the added cholesterol was not incorporated into the lipoproteins in a physiological way. However, we could not find other appropriate ways to investigate interference. Therefore, these results must be confirmed by other methods such as highperformance liquid chromatography. Third, the methods for assessing serum total cholesterol levels were different among 2012 and 2016.

In conclusion, we discovered a strong relationship between serum cholesterol levels and the ratio of direct to total bilirubin. We need to be careful about the concealed relationship when measuring direct bilirubin.

\section{Acknowledgments}

We would like to thank Dr. Yasuo Ido of the Department of Internal Medicine at the National Defense Medical College for analytical support.

Conflicts of Interest

None.

\section{Sources of funding}

This work was supported by a grant-in-aid for scientific research from the Ministry of Education, Science, and Culture of Japan (to MN) (17K09565).

\section{References}

1. Stocker R, Yamamoto Y, McDonagh AF, Glazer AN, Ames BN. Bilirubin is an antioxidant of possible physiological importance. Science 1987; 235: 1043-6.

2. Fujii M, Inoguchi T, Sasaki S, Maeda Y, Zheng J, Kobayashi K, et al. Bilirubin and biliverdin protect rodents against diabetic nephropathy by downregulating $\mathrm{NAD}(\mathrm{P}) \mathrm{H}$ oxidase. Kidney Int 2010; 78 : 905-19.

3. Vitek L. Bilirubin and Atherosclerotic Diseases. Res Physiol 2017; 66: S11-20.

4. Nano J, Muka T, Cepeda M, Voortman T, Dhana K, Brahimaj A, et al. Association of circulating total bilirubin with the metabolic syndrome and type 2 diabetes: a systematic review and metaanalysis of observational evidence. Diabetes Metab 2016; 42: 38997.

5. Maruhashi T, Soga J, Fujimura N, Idei N, Mikami S, Iwamoto Y, et al. Hyperbilirubinemia, augmentation of endothelial function, and decrease in oxidative stress in Gilbert syndrome. Circulation 2012; 126: 598-603.

6. Lin JP, O'Donnell CJ, Schwaiger JP, Cupples LA, Lingenhel A, Hunt SC, et al. Association between the UGT1A1*28 allele, bilirubin levels, and coronary heart disease in the Framingham Heart Study. Circulation 2006; 114: 476-1481.

7. Tanaka M, Budhathoki S, Hirata A, Morita M, Kono S, Adachi M, et al. Behavioral and clinical correlates of serum bilirubin concentrations in Japanese men and women. BMC Endocr Disord 2013; 13: 39. doi: 10.1186/1472-6823-13-39.

8. Nascimento $\mathrm{H}$, Alves AI, Coimbra S, Catarino C, Gomes D, Bronze-da-Rocha E, et al. Bilirubin is independently associated with oxidized LDL levels in young obese patients. Diabetol Metab Syndr 2015; 7: 4.

9. Oda E. Cross-sectional and longitudinal associations between serum bilirubin and dyslipidemia in a health screening population. Atherosclerosis 2015; 239: 31-7.

10. Oda E. A decrease in total bilirubin predicted hyper-LDL cholesterolemia in a health screening population. Atherosclerosis 2014; 235: 334-8.

11. Dullaart RP, de Vries R, Lefrandt JD. Increased large VLDL and small LDL particles are related to lower bilirubin in Type 2 diabetes mellitus. Clin Biochem 2014; 47: 170-5.

12. Zhong P, Sun DM, Wu DH, Li TM, Liu XY, Liu HY. Serum total bilirubin levels are negatively correlated with metabolic syndrome in aged Chinese women: a community-based study. Braz J Med Biol Res 2017; 50: e5252.

13. Karadag F, Sengul CB, Enli Y, Karakulah K, Alacam H, Kaptanoglu B, et al. Relationship between Serum Bilirubin Levels and Metabolic Syndrome in Patients with Schizophrenia Spectrum Disorders. Clin Psychopharmacol Neurosci 2017; 15: 153-62.

14. Lin LY, Kuo HK, Hwang JJ, Lai LP, Chiang FT, Tseng CD, et al. Serum bilirubin is inversely associated with insulin resistance and metabolic syndrome among children and adolescents. Atherosclerosis 2009; 203: 563-8.

15. Giral P, Ratziu V, Couvert P, Carrié A, Kontush A, Girerd X, et al. Plasma bilirubin and gamma-glutamyltransferase activity are inversely related in dyslipidemic patients with metabolic syndrome: relevance to oxidative stress. Atherosclerosis 2010; 210: 607-13.

16. Wang S, Zhang J, Zhu L, Song L, Meng Z, Jia Q, et al. Association between liver function and metabolic syndrome in Chinese 
men and women. Sci Rep 2017; 7: 44844. doi: 10.1038/srep 44844.

17. Lee YB, Lee SE, Jun JE, Jee JH, Bae JC, Jin SM, et al. Change in Serum Bilirubin Level as a Predictor of Incident Metabolic Syndrome. PLoS One 2016; 11: e0168253. doi: 10.1371/journal. pone.0168253.

18. Perlstein TS, Pande RL, Beckman JA, Creager MA. Serum total bilirubin level and prevalent lower-extremity peripheral arterial disease: National Health and Nutrition Examination Survey (NHANES) 1999 to 2004. Arterioscler Thromb Vasc Biol 2008; 28: 166-72.

19. Horsfall LJ, Nazareth I, Petersen I. Cardiovascular Events as a Function of Serum Bilirubin Levels in a Large, Statin-Treated Cohort. Circulation 2012; 126: 2556-64.

20. Zhu B, Wu X, Bi Y, Yang Y. Effect of bilirubin concentration on the risk of diabetic complications: A meta-analysis of epidemiologic studies. Sci Rep 2017; 7: 41681. doi: 10.1038/srep41681.

21. Hopkins PN, Wu LL, Hunt SC, James BC, Vincent GM, Williams RR. Higher serum bilirubin is associated with decreased risk for early familial coronary artery disease. Arterioscler Thromb Vasc Biol 1996; 16: 250-5.

22. Ong KL, Allison MA, Cheung BM, Wu BJ, Barter PJ, Rye KA. The relationship between total bilirubin levels and total mortality in older adults: the United States National Health and Nutrition Examination Survey (NHANES) 1999-2004. PLoS One 2014; 9: e 94479. doi: 10.1371/journal.pone.0094479.

23. Dasgupta A, Sepulveda JL. Accurate results in the clinical laboratory. In: A guide to error detection and correlation. Waltham, MA: Elsevier; 2013. p. 122-6.

24. Fevery J. Bilirubin in clinical practice: a review. Liver Int 2008; 28: 592-605.

25. Miyake Y, Yasunaka T, Ikeda F, Takaki A, Nouso K, Yamamoto K. Fulminant hepatitis: Who survives without liver transplantation? Hepatol Res 2012; 42: 60-7.

26. Executive Summary of The Third Report of The National Cholesterol Education Program (NCEP) Expert Panel on Detection, Evaluation, And Treatment of High Blood Cholesterol In Adults (Adult Treatment Panel III). JAMA 2001; 285: 2486-97.

27. Haffner SM, Miettinen H, Stern MP. The homeostasis model in the San Antonio Heart Study. Diabet Care 1997; 20: 1087-92.

28. Hwang HJ, Kim SH. Inverse relationship between fasting direct bilirubin and metabolic syndrome in Korean adults. Clin Chim Acta 2010; 411: 1496-501.
29. Chang Y, Ryu S, Zhang Y, Son HJ, Kim JY, Cho J, et al. A cohort study of serum bilirubin levels and incident non-alcoholic fatty liver disease in middle aged Korean workers. PLoS One 2012; 7: e37241. doi: 10.1371/journal.pone.0037241.

30. Karadag F, Sengul CB, Enli Y, Karakulah K, Alacam H, Kaptanoglu B. Relationship between Serum Bilirubin Levels and Metabolic Syndrome in Patients with Schizophrenia Spectrum Disorders. Clin Psychopharmacol Neurosci 2017 May 31; 15: 153-62.

31. Kurosaka K, Senba S, Tsubota H, Kondo H. A new enzymatic assay for selectively measuring conjugated bilirubin concentration in serum with use of bilirubin oxidase. Clin Chim Acta 1998; 269: 125-36.

32. Devgun MS, Richardson C. Direct bilirubin in clinical practice Interpretation and haemolysis interference guidance reassessed. Clin Biochem 2016; 49: 1351-3.

33. Gautam A, Seligson H, Gordon ER, Seligson D, Boyer JL. Irreversible Binding of Conjugated Bilirubin to Albumin in Cholestatic Rats. J Clin Invest 1984; 73: 873-7.

34. Dhungana N, Morris C, Krasowski MD. Operational impact of using a vanadate oxidase method for direct bilirubin measurements at an academic medical center clinical laboratory. Pract Lab Med 2017; 8: 77-85.

35. Whitmer DI, Russell PE, Ziurys JC, Gollan JL. Hepatic Microsomal Glucuronidation of Bilirubin Is Modulated by the Lipid Microenvironment of Membrane-bound Substrate. J Biol Chem 1986; 261: 7170-7.

36. Szabó M, Veres Z, Bátai-Konczos A, Kékesi O, Kis E, Szabó K, et al. Statins alter the hepatobiliary transport of unconjugated and conjugated bilirubin in sandwich-cultured rat hepatocytes. Toxicol In Vitro 2014; 28: 1136-43.

37. Ramjiganesh T, Roy S, Freake HC, McIntyre JC, Fernandez ML. Corn fiber oil lowers plasma cholesterol by altering hepatic cholesterol metabolism and up-regulating LDL receptors in guinea pigs. J Nutr 2002; 132: 335-40.

38. Li T, Chiang JY. Bile acid signaling in metabolic disease and drug therapy. Pharmacol Rev 2014; 66: 948-83.

39. Bertolotti M, Crosignani A, Del Puppo M. The use of stable and radioactive sterol tracers as a tool to investigate cholesterol degradation to bile acids in humans in vivo. Molecules 2012; 17: 193968.

40. Van Erpecum KJ. Pathogenesis of cholesterol and pigment gallstones: an update. Clin Res Hepatol Gastroenterol 2011; 35: 2817. 